\title{
Performance Evaluation of Flocking-Based Distributed Cyber-Physical Control for Smart Grid
}

\author{
Abdallah K. Farraj* Eman M. Hammad* Jin Wei* Deepa Kundur* Karen L. Butler-Purry ${ }^{\dagger}$ \\ ${ }^{*}$ Department of Electrical and Computer Engineering, University of Toronto, Toronto, Ontario, Canada \\ $\dagger$ Department of Electrical and Computer Engineering, Texas A\&M University, College Station, Texas, USA \\ Email: \{abdallah, ehammad, jinwei, dkundur\}@ece.utoronto.ca, klbutler@tamu.edu
}

\begin{abstract}
A flocking-based control strategy has recently been proposed to stabilize power systems during transient instability. The flocking paradigm offers a rich understanding of the coupling and interactions between the cyber and physical system components and employs this insight to build more intelligent distributed control schemes. This article investigates the performance of flocking-based control in the presence of nonideal practical measurement, communication and storage constraints. We identify intrinsic design tradeoffs that exist between measurement parameters and communication specifications to achieve a specific level of controller performance.
\end{abstract}

\section{INTRODUCTION}

Interest in smart grid system development has surged recently from a variety of stakeholders ranging from device vendors, utilities and academic researchers. Smart grid systems integrate advanced sensor technologies, communication systems and control to help improve the efficiency, reliability and resilience of power generation, transmission and delivery. The two way information and power flow facilitated in smart grid systems enables utility companies to better manage and control energy resources and customer demand. Moreover, it facilitates the integration of renewable energy sources and storage to aid in decreasing the nation's carbon footprint.

More recently, the smart grid has been viewed as a cyberphysical system comprised of the interaction of a communication and information network with a power delivery grid. The associated cyber assets include communication infrastructure, sensors and metering, computing systems and advanced distributed control systems. The physical components include typical power delivery components including generators, transmission lines, transformers and substation components among others. The physical-to-cyber interfaces are at sensors and measurement devices while the cyber-to-physical junctions occur at the controllers that consume data and actuate change on the delivery system.

It is well known that the greater dependence of power delivery on cyber technology leads to potential vulnerabilities stemming from cyber attack [1], [2]. Attacks on the communication and information systems prevalent in today's information technology systems, will propagate across the cyber-tophysical bridge and result in cascading failures eventually on the power delivery system. Thus distributed control represents an opportunity to help mitigate the propagation and impact of cyber attacks on smart grid systems by broadening the possible approaches for protection.
Classically a defense-in-depth approach was proposed for security in which information protection metrics were employed throughout the various layers of an information system to provide protection from a variety of perspectives. We argue that for cyber-physical smart grid systems in addition to defense-in-depth a defense-in-breath approach also serves valuable. Specifically, we argue of the need for distributed control paradigms to improve system resilience to disturbance especially those resulting from cyber attack. The performance of such control paradigms must be carefully studied in the context of practical limitations to understand the potential opportunities to mitigate attacks effectively.

Recently, a nonlinear flocking-based controller has been proposed by Wei et al. [3]-[6] for transient stabilization of a smart grid system in the face of disturbances such as those stemming from cyber attack. Thus, in this paper we focus on the performance evaluation of the flocking-based controller for smart grid systems in terms of a number of practical cyber and physical constraints. We then aim to glean insights on performance tradeoffs for general distributed control paradigms.

Section II provides an overview of the flocking paradigm for distributed control. Section III presents the system upon which we conduct the performance evaluation. A performance evaluation is conducted in Section IV followed by final remarks in Section V.

\section{Flocking-BASEd SMART GRID Distributed CONTROL}

In essence, flocking represents an aggregate behavior amongst entities to achieve a shared group behavior. Recently, flocking theory has been employed to develop an framework that equates transient stability of synchronous generators to the behavior of bird-like objects forming a flock [3]-[6].

\section{A. Cyber-Physical Framework}

We consider a multi-agent framework that incorporates both cyber and physical elements of a smart grid system. Figure 1 presents one such system applicable to the 3-Generator 9-bus WECC system considered in this paper (see Figure 2), but the paradigm scales in a straightforward way to larger systems. Each cyber-physical agent consists of: (1) a synchronous generator, (2) a sensor such as a phasor measurement unit (PMU) that acquires data from the state of the generator 


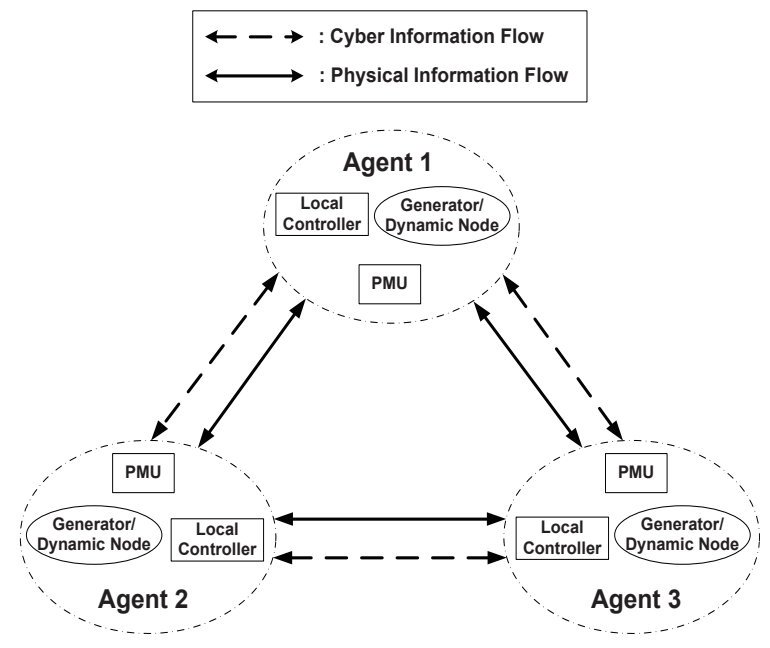

Fig. 1. Equivalent networked multi-agent dynamic system model.

including rotor phase angle and frequency, and (3) a local distributed controller that obtains information from sensors (of its own agent and others) to compute a control signal that is applied via an energy storage system (ESS) to the synchronous generator bus. The local controller and sensor are considered to be a cyber elements due to their data acquisition, communications and computation tasks. The synchronous generator and ESS are considered to be physical components. The state of an agent is considered to be the state of its corresponding synchronous generator.

Our focus is on the transient stability problem [7]. Therefore, one objective of the local controller is to achieve generator frequency synchronization in the face of cyber-physical disturbance. As such, the local controllers make use of fastacting external power sources in order to achieve system balance. These sources in practice include battery storage devices, plug-in hybrid electric vehicles (PHEVs) and flywheels, and may be separate for each agent. For convenience, sensor data is modeled as being sent to a data concentrator such that all agents can access the information to make effective control decisions. The reader should note that models in which sensors directly communicate with one another would not change the paradigm. In fact, Fig. 1 presents the equivalent multi-agent model considering the effective information (cyber) and power (physical) flows.

\section{B. Transient Stability}

It is well-known that transient stability describes the ability of a power system to remain in synchronism when subjected to large disturbances, such as transmission line faults and generator loss [7]. Achieving transient stability in the presence of a cyber attack disturbance or fault consists of maintaining both exponential frequency synchronization and phase angle cohesiveness through possible application of control strategies. Exponential frequency synchronization requires the frequencies of all the generators agree asymptotically with a common value $f_{0}$ typically set to $60 \mathrm{~Hz}$ and normalized in this formulation to 1 . Phase angle cohesiveness necessitates that the difference between the phase angle of each generator and the center of inertia (COI) phase (defined below) should be below a pre-defined threshold typically chosen to be $5 \pi / 9$ [8]. Analytically, we can write these goals as:

1) Exponential frequency synchronization:

$$
\omega_{i}(t) \rightarrow 0 \text {, as } t \rightarrow \infty
$$

where $\omega_{i}(t)$ is the normalized frequency of Generator $i$.

2) Phase angle cohesiveness:

$$
\left|\delta_{i}(t)-\delta_{C O I}(t)\right| \leq \gamma, \forall t
$$

where $\delta_{i}(t)$ is the rotor angle of Generator $i, \gamma$ is normally set to $5 \pi / 9$ in practice [8], and $\delta_{C O I}(t)$ is the COI phase angle defined as:

$$
\delta_{C O I}=\frac{\sum_{i=1}^{N} M_{i} \delta_{i}}{\sum_{i=1}^{N} M_{i}} .
$$

where $M_{i}$ represents generator inertia as we discuss in the next section.

\section{Nonlinear Flocking-Based Distributed Control}

For flocking-based control, generator state trajectory analogies are made for the following heuristic interaction rules for flocks [9]:

1) Flock Centering: agents attempt to stay close to nearby flockmates,

2) Collision Avoidance: agents avoid collisions with nearby flockmates,

3) Velocity Matching: agents attempt to match velocity with nearby flockmates.

It is observed that that flock centering and velocity matching are analogous to the phase angle cohesiveness and the exponential frequency synchronization objectives of the transient stability problem, respectively [4]. This analogy inspires us to reformulate the problem of transient stability as a task of flocking formation control. The use of flocking theory provides a strong theoretical foundation for stability and to develop insights under model assumptions and we believe provides some robustness as exhibited by flocks in nature. Moroever, the analogy enables interpretation of the control paradigm as requiring the use of distributed fast acting sources or ESSs. The local control and ESS are at the cyber-to-physical bridge to aid in system resilience.

The flocking-based controller for each agent is nonlinear and employs synchronized sensor information from its own generator and neighboring generators to facilitate transient stabilization. Recent work has focused on a hierarchical approach for selectively employing distributed control and sensor data [4]. The reader should note that we employ the nonhierarchical approach for the studies in this paper to better understand the strong dependencies between the cyber and physical domains. Although the prior work sheds light on the application of flocking control for emerging power systems, a more comprehensive assessment as to the performance of such control in the presence of non-ideal conditions such as communication delay and noise is needed. 
Thus, this work considers the impact of practical limitations on the performance of distributed control. Specifically, we explore the performance of flocking control on the WECC 3-generator 9-bus power system. Specifically, we focus on the effects of:

- Communication latency between the sensors and the controllers: the communication network is often idealized as having zero delay. In practice, communication systems especially those envisioned for future power systems will have some delay that we consider in our tests;

- Sensor sampling frequency: the physical-to-cyber bridge involves conversion of continuous-time signals into digital signals for processing by a computer. There is a sampling frequency associated with the analog-todigital conversion that we consider;

- Quantization-level: the analog-to-digital conversion also includes quantization of the amplitude of the physical signals that is included in this assessment;

- Signal-to-noise ratio (SNR) at the sensor: most approaches to controller design do not account for nonideals of the measurement process such as noise; we consider the effects of fundamental noise floor in the signal acquisition process;

- Limits on the external power employed by the controller: although stability is theoretically proven for general control signals, in practice there is a maximum absolute power limit on the power that can be produced or absorbed by the ESS. In our studies this is modeled as clipping; and

- Outage probability of the channel between the sensors and the controller: denial-of-service of communications can have profound impacts on operation; we study the effects of communication outage on system stabilization.

We evaluate controller performance in terms of both external power usage and generator stability time. The former metric represents a measure of the effort and overhead required to stabilize the system whereas the latter provides an idea of the time scale for system stabilization. If the time for stabilization is too long, then equipment may be at risk of damage and an opportune window may be available for an attacker to create further havoc on an already vulnerable system.

\section{Evaluation Case Study System}

We consider the effects of non-ideal conditions on distributed control in the context of the WECC 3-generator 9-bus power system as shown in Fig. 2. We employ a power system of this fundamental scale to better understand the underlying cyber-physical interactions that are affected by practical communication and measurement limitations. As discussed in Section II each synchronous generator is set up with a local controller and actuating ESS.

In the power system of Fig. 2, Bus 1 is assigned as the slack bus, Buses 2 and 3 are PV buses, and Buses 7 and 8 are PQ (load) buses. The parameters of the power system are shown

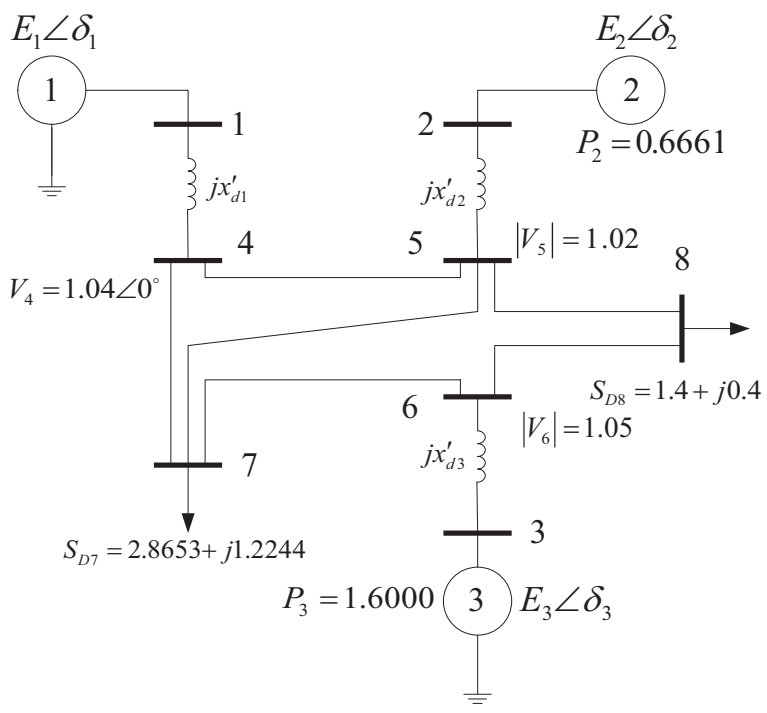

Fig. 2. WECC power system

\begin{tabular}{|c|l|}
\hline Parameter & Description \\
\hline$t$ & independent time variable \\
$N$ & number of generators in the power system \\
$E_{i}$ & internal voltage of Generator $i, i=1, \ldots, N$ \\
$P_{e, i}$ & electrical power of Generator $i$ \\
$P_{m, i}$ & mechanical power of Generator $i ; P_{m, i}>0$ \\
$M_{i}$ & inertia of Generator $i ; M_{i}>0$ \\
$D_{i}$ & damping constant of Generator $i ; D_{i}>0$ \\
$\delta_{i}$ & rotor angle of Generator $i$ \\
$\omega_{i}$ & relative rotor frequency of Generator $i$ \\
$G_{i i}$ & equivalent shunt conductance of Generator $i ; G_{i i} \geq 0$ \\
$Y_{i k}$ & Kron-reduced equivalent admittance between Gen. $i$ and $k$ \\
\multicolumn{2}{c}{ SySTEM PARAMETERS } \\
\hline
\end{tabular}

in Table I, and are in per unit values, except for $M_{i}$ and $D_{i}$ which are expressed in seconds.

As discussed in [3], the swing equation model of a synchronous generator, effective for studying transient stability, is employed for development of the controller. We assume that the system parameters remain constant from the time of the disturbance and through the possible destabilization process. Let $\dot{\delta}_{i}$ and $\dot{\omega}_{i}$ denote the derivatives of the Generator $i$ rotor angle $\delta_{i}$ and frequency $\omega_{i}$ with respect to time, respectively, then the swing equation of an interconnected power system is expressed as [10], [11]:

$$
\begin{aligned}
& \dot{\delta}_{i}=\omega_{i} \\
& \dot{\omega}_{i}=-\frac{D_{i}}{M_{i}} \omega_{i}+\frac{1}{M_{i}}\left(P_{m, i}-P_{e, i}\right),
\end{aligned}
$$

where the electrical power of Generator $i$ is defined as

$$
P_{e, i}=\sum_{k=1}^{N}\left|E_{i}\right|\left|E_{k}\right|\left(G_{i k} \cos \left(\delta_{i}-\delta_{k}\right)+B_{i k} \sin \left(\delta_{i}-\delta_{k}\right)\right),
$$

where $G_{i k}=G_{k i} \geq 0$ is the Kron-reduced equivalent conductance between Generators $i$ and $k, B_{i k}=B_{k i}>0$ is 
the Kron-reduced equivalent susceptance between Generators $i$ and $k$, and $Y_{i k}=G_{i k}+\sqrt{-1} B_{i k}$ is the Kron-reduced equivalent admittance between Generators $i$ and $k$. All of $Y_{i k}, G_{i k}$, and $B_{i k}$ are expressed in per unit values. Using $\phi_{i k}=\arctan \left(\frac{G_{i k}}{B_{i k}}\right)$ and $P_{i k}=\left|E_{i}\right|\left|E_{k}\right|\left|Y_{i k}\right|$, the swing equation is reduced to:

$$
\begin{aligned}
\dot{\delta}_{i}= & \omega_{i} \\
\dot{\omega}_{i}= & -\frac{D_{i}}{M_{i}} \omega_{i}+\frac{1}{M_{i}}\left(P_{m, i}-\left|E_{i}\right|^{2} G_{i i}\right) \\
& -\frac{1}{M_{i}} \sum_{k=1, k \neq i}^{N} P_{i k} \sin \left(\delta_{i}-\delta_{k}+\phi_{i k}\right) .
\end{aligned}
$$

As described in Eqs. (4) and (6), the dynamics generators will not stabilize easily in the presence of severe disturbance. However, as first introduced in [3] applying an external power injection or absorption signal at each generator, denoted $U_{i}$, alters the swing equation to:

$$
\begin{aligned}
\dot{\delta}_{i}= & \omega_{i} \\
\dot{\omega}_{i}= & -\frac{D_{i}}{M_{i}} \omega_{i}+\frac{1}{M_{i}}\left(P_{m, i}-\left|E_{i}\right|^{2} G_{i i}\right) \\
& -\frac{1}{M_{i}} \sum_{k=1, k \neq i}^{N} P_{i k} \sin \left(\delta_{i}-\delta_{k}+\phi_{i k}\right)+\frac{1}{M_{i}} U_{i} .
\end{aligned}
$$

In flocking-based control, $U_{i}$ can be used to achieve both frequency synchronization and phase cohesiveness between the synchronous generators. Specifically, $U_{i}$ is calculated as [3]:

$$
\boldsymbol{U}=f(\boldsymbol{\delta}, \boldsymbol{\omega}),
$$

where

$$
\begin{aligned}
f(\boldsymbol{\delta}, \boldsymbol{\omega})= & \sum_{k=1, k \neq i}^{N}\left[\int_{t_{0}}^{t} \rho\left(\delta_{i}-\delta_{k}\right) \mathrm{d} t\right] \mathbf{1} \\
& -\boldsymbol{G} \boldsymbol{\delta}-\boldsymbol{B} \boldsymbol{\omega} \cdot \boldsymbol{D}-c\left(\boldsymbol{\delta}-\boldsymbol{\delta}_{\mathbf{0}}\right),
\end{aligned}
$$

and $\boldsymbol{U}=\left[U_{1}, U_{2}, \ldots, U_{N}\right]^{T}$ (where $[\cdot]^{T}$ is the transpose matrix operator), $t_{0}$ is the time to activate the flocking control, $t$ is the time to calculate the value of the control, $\rho$ is a control parameter, $c$ is a navigation term, $\boldsymbol{\delta}_{\mathbf{0}}=\left[\delta_{0_{1}}, \delta_{0_{2}}, \ldots, \delta_{0_{N}}\right]^{T}$ are constant terms, $\boldsymbol{D}=\left[D_{1}, D_{2}, \cdots, D_{M}\right]^{T}, \boldsymbol{\delta}=\left[\delta_{1}, \delta_{2}, \cdots, \delta_{N}\right]^{T}$, and $\boldsymbol{\omega}=\left[\omega_{1}, \omega_{2}, \cdots, \omega_{N}\right]^{T}$. Moreover, $\boldsymbol{B}$ and $\boldsymbol{G}$ are cyber control matrices.

It is noted that the swing-equation based model of the local generators does not include governor control. The presence of the governor control, which operates at a much slower time scale than the flocking controller, we assert will only aid in improving the transient stabilization of the system. Thus, our analysis is more conservative assessment of system performance.

\section{Performance of the Flocking-Based CONTROLLER}

We consider the performance of flocking-based control in terms of stability time and average external power necessary for stabilization on the 3-generator 9-bus WECC system of Fig. 2. As shown in Fig. 3, $N=3$ for the test system. We let $D_{1}=0.005$ seconds, $D_{2}=0.001$ seconds, $D_{3}=0.002$ seconds, $X_{d 1}^{\prime}=0.08 \mathrm{pu}, X_{d 2}^{\prime}=0.18 \mathrm{pu}, X_{d 3}^{\prime}=0.12 \mathrm{pu}$, $M_{1}=50 \mathrm{msec}, M_{2}=15 \mathrm{msec}$, and $M_{3}=35 \mathrm{msec}$.

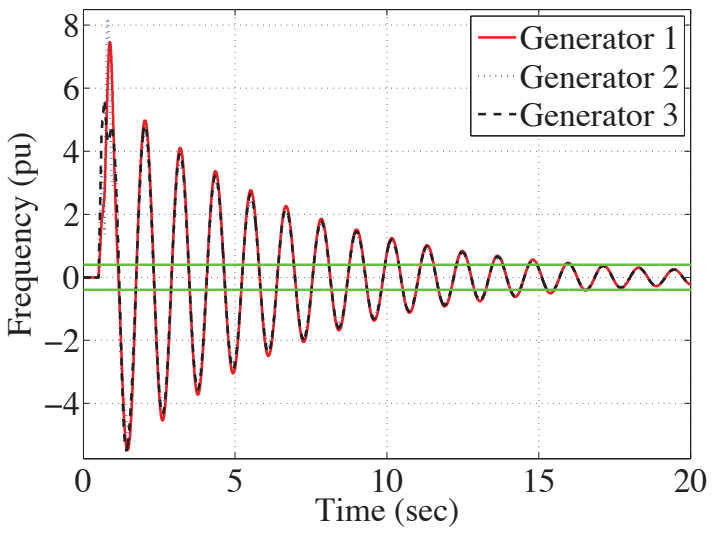

Fig. 3. Relative frequency for the system generators

The power system is assumed to be running normally from $t=0$ to $t=0.5$ seconds. Then, a 3-phase fault occurs at Bus 6 at $t=0.5$ seconds. Line 6-7 is tripped out to clear the fault at $t=0.6$ seconds. Finally, the flocking controller is initiated on all three generator agents from $t=0.7$ seconds to $t=70$ seconds.

Running load flow analysis for the power system prior to the occurrence of the 3-phase fault yields $E_{1}=1.162 \angle 7.605^{\circ}$ pu, $E_{2}=1.261 \angle-8.904^{\circ} \mathrm{pu}$, and $E_{3}=1.244 \angle-4.024^{\circ}$ $\mathrm{pu}$. Because the system is balanced and there are no prefault transients, the mechanical power equals the electrical power for each synchronous generator. Consequently, and using Eq. (5), the values of the mechanical power for each generator are calculated as:

$$
\begin{aligned}
& P_{m, 1}=1.9989 \mathrm{pu} \\
& P_{m, 2}=0.6661 \mathrm{pu} \\
& P_{m, 3}=1.6000 \mathrm{pu} .
\end{aligned}
$$

The simulation results are conducting using MATLAB/Simulink where the simulation run time is 70 seconds. The stability time of a generator is found by determining the time after which the normalized frequency of the generator is restricted to some threshold. Fig. 3 displays the relative frequencies of the three generators; it is noted that when the threshold is set to $0.4 \mathrm{pu}$, the three generators need slightly more than 16 seconds to stabilize.

\section{A. Sensor Sampling Frequency}

The sensor state readings for the rotor angles $\delta_{i}$ and frequencies $\omega_{i}$ for $i=1,2,3$ must be sampled prior to transmission across the communication channel. Assuming the sampling frequency is denoted $f_{s}$ with corresponding sampling period $T_{s}=\frac{1}{f_{s}}$, we note that higher values of $f_{s}$ (i.e., lower values of $T_{s}$ ) imply that the sensors are tracking the system states more frequently. Consequently, as $T_{s}$ becomes lower, the controller obtains more frequent state information updates. Mathematically, $\boldsymbol{U}$ is represented as $\boldsymbol{U}(t)=f\left(\boldsymbol{\delta}\left(n T_{s}\right), \boldsymbol{\omega}\left(n T_{s}\right)\right)$ for $n T_{s} \leq t<(n+1) T_{s}$ and $n=1,2, \ldots$

Fig. 4 shows the performance of the distributed control versus sampling period. As the sampling rate is lower, the 

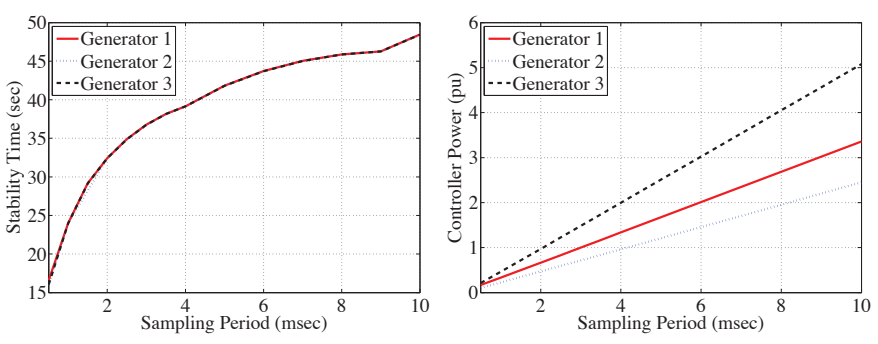

Fig. 4. Performance against sampling period
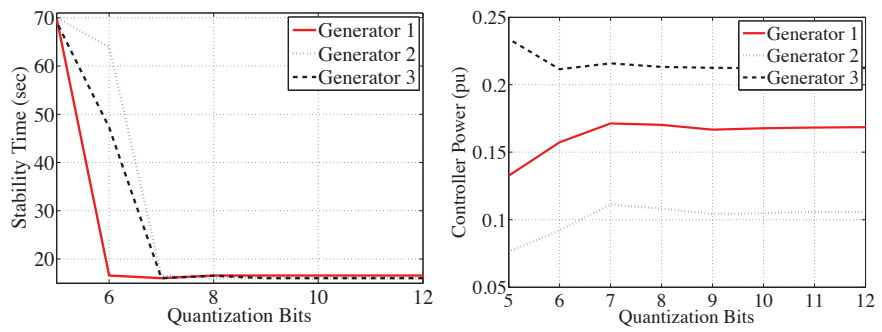

Fig. 5. Performance against number of quantization bits

time to stabilization increases. For example, it takes more than 30 seconds to achieve transient stability if $f_{s} \leq 600 \mathrm{~Hz}$. Furthermore, with increasing $T_{s}$, the control requires higher external power. As the sensors' updates are less frequent, the deviation between the system parameters and the output of the controller increases; consequently, the controller requires more power to compensate the for higher difference.

\section{B. Quantization}

Quantization of the sensor readings is another processing stage during the measurement process. The goal of quantization is to discretize a signal amplitude prior to bit encoding. As the bit depth (the number of quantization bits) increases, the granularity of quantization increases and quantization error decreases. As the sensors use more quantization bits, the quantization levels increase and the quantization noise decreases. In this case, $\boldsymbol{U}(t)=f(Q(\boldsymbol{\delta}(t)), Q(\boldsymbol{\omega}(t)))$ where $Q(\cdot)$ is the appropriate quantization function.

Fig. 5 presents the effects of signal quantization on the performance of the flocking controller. A coarse representation of the sensor readings (with a smaller bit depth) negatively affects the controller's performance; for example, the power system is not stabilized if the sensors use a bit depth below 5 . However, if the sensors use a bit depth of 7 bits (or more), the flocking-based controller can stabilize the three generators in less than 20 seconds.

\section{Communication Latency}

The communication latency is the aggregate result of various processing and propagation delays in the communication network. It is a function of the communication medium, network and protocols employed. Delays in smart grid communications are typically a result of sensor sampling and quantization delay, possible cryptographic delay if security processing
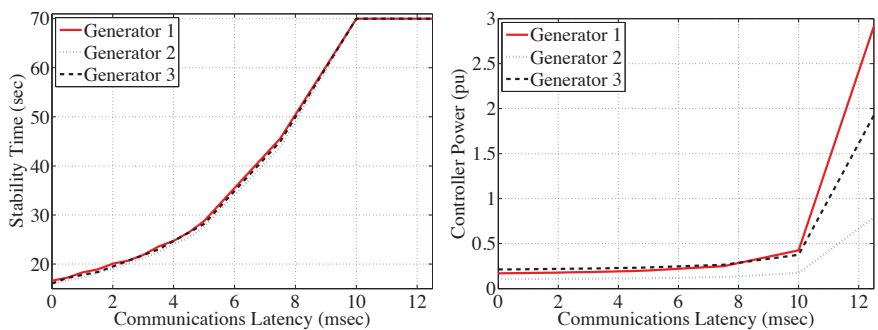

Fig. 6. Performance against communication latency
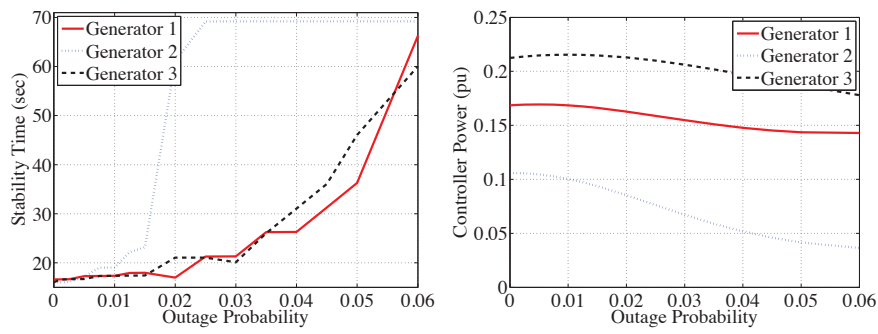

Fig. 7. Performance against outage probability

is employed, channel propagation delay, and queueing delay. Latency can be variable or fixed for each data transmission. The case of fixed latency is considered in this work as it is consistent with the notion that sensor data is assigned by aggregators in smart grid communication networks. Mathematically, we have $\boldsymbol{U}(t)=f(\boldsymbol{\delta}(t-\tau), \boldsymbol{\omega}(t-\tau))$ where $\tau$ is the fixed communication latency.

Fig. 6 displays the effects of the communication latency on the controller performance. It is noted that as the generator state sensor data takes a longer time to arrive to the controller, both the stability time and the external power increase. Further, when the communication latency is higher than $10 \mathrm{msec}$, the system generators cannot be stabilized by the controller within the 70 -second run time.

\section{Channel Outage Probability}

Channel outages occur when the transmission rate on the communication link between the sensors and the controller exceeds the instantaneous capacity of that channel. Thus, when an outage occurs, the receiver (i.e., the controller) cannot decode the transmitted data correctly. Consequently, generator state information is not available to the controller during a channel outage. In this case, we employ the following model for the control signal $\boldsymbol{U}(t)=f(\boldsymbol{\delta}(t), \boldsymbol{\omega}(t))$ only when the communication channel experiences no outage and $\boldsymbol{U}(t)=0$ otherwise.

Fig. 7 shows the performance of the controller against the outage probability. As one expects, the stability time increases as the channel experiences more outages; however, it is interesting to note that when the communication channel experiences outages with a probability of $8 \%$ or more, the controller does not stabilize the power system during the simulation run time. 

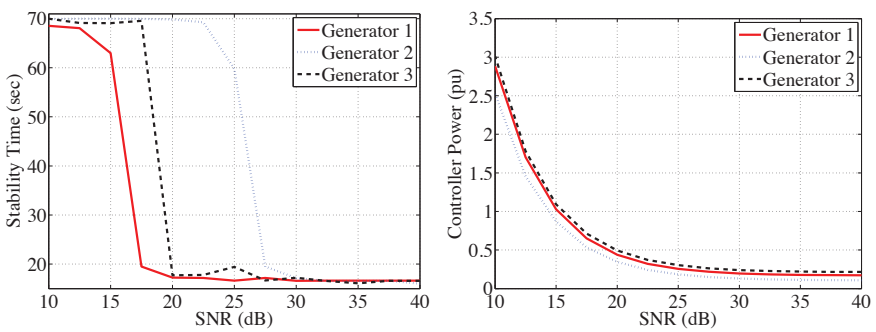

Fig. 8. Performance against signal-to-noise ratio
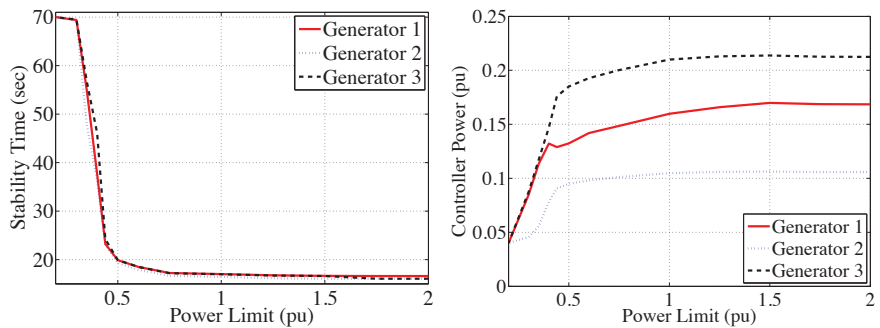

Fig. 9. Performance against power limit

\section{E. Signal-to-Noise Ratio}

The SNR given by: SNR $=10 \log _{10}\left(\frac{E_{S}}{E_{N}}\right)$ where $E_{S}$ is the energy of the original uncorrupted signal, $E_{N}$ is the energy of the associated noise which is computed as the deviation between the original signal at the source and the version received at the controller, and SNR is measured in units of $\mathrm{dB}$. Thus SNR is a measure of the quality of the received sensor signal at the controller; the higher the value of SNR, the higher the fidelity and accuracy. If the measured noise signals are represented as $\boldsymbol{n}_{\delta}$ and $\boldsymbol{n}_{\omega}$, then $\boldsymbol{U}(t)=f\left(\boldsymbol{\delta}(t)+\boldsymbol{n}_{\boldsymbol{\delta}}(t), \boldsymbol{\omega}(t)+\boldsymbol{n}_{\boldsymbol{\omega}}(t)\right)$.

Fig. 8 displays the effect of the SNR on the performance of the flocking controller. It is noted that a high value of SNR is required by the controller in order to stabilize the power system. For a SNR value of less than about $17.5 \mathrm{~dB}$, two of the three generators cannot be stabilized. Further, as the SNR value increases, the flocking-based controller uses less external power in order to run.

\section{F. External Power Limit}

The flocking controller relies on a fast-acting external power injection and absorption source, $\boldsymbol{U}$, to contribute to the power system dynamics. However, $\boldsymbol{U}$ will have practical physical limitations, specifically, $|\boldsymbol{U}| \leq P_{\max }$. The power limit can be a result of the number and capacity of the used batteries. Consequently, for this case $|\boldsymbol{U}(t)|=\min \left(|f(\boldsymbol{\delta}(t), \boldsymbol{\omega}(t))|, P_{\max }\right)$.

The effects of the external power limit are shown in Fig. 9. It is noted that the controller performs well when the external power supply is not restricted to limits below $0.5 \mathrm{pu}$. For values of $P_{\max } \geq 0.5 \mathrm{pu}$, the flocking controller can effectively stabilize the WECC power system within 20 seconds.

\section{FINAL REMARKS}

The role of distributed control in smart grid systems is expanding to include improving resilience to cyber and physical faults. Thus it is important to assess the effect of practical cyber and physical limitations on controller performance.

A flocking-based control was recently proposed for transient stability of smart grid systems. Through a series of studies we demonstrate the performance of the controller under practical constraints. We believe that the results of this work can be extended to more general distributed controllers and glean the following insights regarding implementing distributed control for protection and resilience.

There appear to be fundamental design trade-offs that must be considered when employing complex control. The sampling period, quantization bit depth and overall measurement SNR must be accounted for when designing an appropriate communication system for smart grid applications. If the associated measurement parameters largely deviate from ideal values then there will be tighter communication latency bounds for a given level of stabilization performance. In addition, it is found that outage probability needs to be fixed below a specific threshold (which was found to be of reasonable value in our studies) and power limit constraints do not severely hinder performance as the power limit bounds appear reasonable.

\section{ACKNOWLEDGMENT}

This work was supported by the Natural Sciences and Engineering Research Council of Canada and the U.S. National Science Foundation under grant ECCS-1028246.

\section{REFERENCES}

[1] S. M. Amin, "Energy infrastructure defense systems," Proceedings of the IEEE, vol. 93, pp. 861-875, May 2005.

[2] S. M. Amin, "For the good of the grid," IEEE Power and Energy Magazine, vol. 6, pp. 48-59, November/December 2008.

[3] J. Wei, D. Kundur, T. Zourntos, and K. Butler-Purry, "A flockingbased dynamical systems paradigm for smart power system analysis," in Proc. IEEE Power \& Energy Society General Meeting, (San Diego, California), July 2012.

[4] J. Wei and D. Kundur, "Two-tier hierarchical cyber-physical security analysis framework for smart grid," in Proc. IEEE Power \& Energy Society General Meeting, (San Diego, California), July 2012.

[5] J. Wei, D. Kundur, and T. Zourntos, "On the use of cyber-physical hierarchy for smart grid security and efficient control," in Proc. IEEE Canadian Conference on Electrical and Computer Engineering (CCECE), (Montreal, Canada), April-May 2012.

[6] J. Wei, D. Kundur, T. Zourntos, and K. Butler-Purry, "Probing the telltale physics: Towards a cyber-pysical protocol to mitigate information corruption in smart grid systems," in Proc. IEEE International Conference on Smart Grid Communications, (Tainan, Taiwan), pp. 372-377, November 2012.

[7] P. Kundur, Power System Stability and Control. McGraw-Hill, 1994.

[8] P. Sauer and M. Pai, Power System Dynamics and Stability. Prentice Hall, 1997.

[9] C. Reynolds, "Flocks, herds, and schools: a distributed behavioral model," Computer Graphics, vol. 21, pp. 25-34, July 1987.

[10] F. Dörfler and F. Bullo, "Synchronization and Transient Stability in Power Networks and Non-Uniform Kuramoto Oscillators," in American Control Conference (ACC), pp. 930-937, 2010.

[11] P. M. Anderson and A. A. Fouad, Power System Control and Stability. IEEE Power Systems Engineering Series, Piscataway, NJ: IEEE Press, 1994. 